\title{
Discovery of the Philadelphia chromosome: a personal perspective
}

\author{
Peter C. Nowell \\ Department of Pathology and Laboratory Medicine, University of Pennsylvania School of Medicine, Philadelphia, Pennsylvania, USA.
}

\begin{abstract}
Almost 50 years ago, David Hungerford and I noticed an abnormally small chromosome in cells from patients with chronic myelogenous leukemia (CML). This article is a personal perspective of the events leading to the discovery of this chromosome, which became known as the Philadelphia chromosome. As technology advanced over subsequent decades, the translocation resulting in the Philadelphia chromosome has been identified, its role in the development of CML has been confirmed, and a therapy directed against the abnormal protein it produces has shown promising results in the treatment of patients with CML.
\end{abstract}

\section{Early forays into tumor genetics}

In the latter part of the nineteenth century, several German pathologists observed gross mitotic abnormalities in tissue sections of many different human malignancies and suggested that abnormalities such as these might have an important role in the initiation and development of human tumors (1). This was very shortly after the terms "chromosome" and "mitosis" were first introduced. Several decades later, in 1914, the biologist Theodore Boveri generated several hypotheses about the role of somatic genetic alterations in the development of cancer (2). His central hypothesis - that mammalian tumors might be initiated by mitotic abnormalities that resulted in a change in the number of chromosomes in a cell (aneuploidy) - was based on his observation that mitotic abnormalities in sea urchins often led to the abnormal development of the organism (2). This hypothesis was then extended to include other concepts, for example, that tumor cell populations are genetically unstable; that tumors originate from a single cell; that tumor cells become unresponsive to external growth regulation because they either gain or lose critical chromosomes; and that tumors might result from genetic alterations that were not visible with a microscope because they did not involve entire chromosomes (2). Although Boveri admitted to having no direct knowledge of cancer, many of these hypotheses were subsequently proven correct. However, proof had to wait for the development of techniques sophisticated enough to test these hypotheses, meaning that they were largely ignored for the next 30 years. Indeed, during this time, techniques for counting and characterizing mammalian chromosomes were so inadequate that when Painter determined in 1921 that humans had 48 chromosomes (3), this observation was confidently accepted. Therefore, it is not surprising that other researchers were reporting the chromosome number of human tumors as normal.

This situation did not change substantially over the next few decades, until chromosome studies of long-established transplantable rodent tumors, using "squash" techniques that had been developed in other species, permitted the demonstration of multiple chromosomal alterations in late-stage neoplasms. Furthermore, the identification of one or more characteristic chro-

Nonstandard abbreviations used: ABL, v-abl Abelson murine leukemia viral oncogene homolog; BCR, breakpoint cluster region; $\mathrm{CML}$, chronic myelogenous leukemia. Conflict of interest: The author has declared that no conflict of interest exists. Citation for this article: J. Clin. Invest. 117:2033-2035 (2007). doi:10.1172/JCI31771. mosome abnormalities in all the cells of a given tumor provided evidence for Boveri's hypothesis that tumors arose from a single genetically altered cell. This concept was initially termed "the stem line concept" but was later developed into the "clonal evolution" model of tumor development. The observation that tumor cells with a specific chromosome abnormality had additional chromosomal variations that differed from cell to cell provided support for the idea proposed by Winge (4) that a series of genetic changes could cause the stepwise clinical and biological progression of tumors. Further evidence for the stem line concept was provided by Makino and Sasaki, who showed that a human tumor could be characterized by a single chromosomal abnormality (5).

Meanwhile, work had begun to investigate non-neoplastic human and other mammalian tissues and to develop improved tissue culture techniques, bringing us into the era of modern cytogenetics. The quality of chromosome preparations was markedly improved by the use of colchicine, which inhibits microtubule assembly and thereby arrests cells in mitosis, and of hypotonic solutions, which disrupt the mitotic spindle and expand the cell (6). This enabled Tjio and Levan to accurately determine that the normal human chromosome number is 46 (7), and this was soon confirmed by Ford and Hamerton (8). The improved cytogenetic methods were also used to study chromosome arrangements in various pathological conditions, and it was found that particular human disorders were associated with specific chromosomal abnormalities (e.g., Down syndrome was shown to be associated with the presence of an extra copy of chromosome 21, and Turner syndrome was shown to be associated with the presence of only a single X sex chromosome). However, only a small number of human tumors, most of which were leukemias, were examined using the new cytogenetic methods, and no specific chromosome alterations were identified. Therefore, Bayreuther, who had investigated various types of tumors, concluded in 1960 that the chromosome complement of most human tumors was normal (9).

\section{Consistent chromosomal alterations in chronic myelogenous leukemia: the discovery of the Philadelphia chromosome}

It was at this point, serendipitously, that I entered the field of tumor cytogenetics. I joined the faculty of the University of Pennsylvania in 1956, having spent two years in the Navy studying radiation carcinogenesis and bone marrow transplantation. I was particularly interested in leukemias and lymphomas because in the 


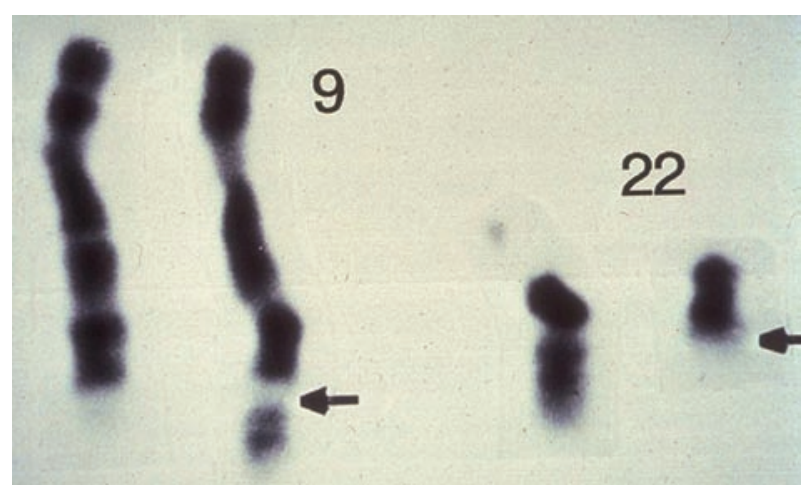

Figure 1

Karyotype of an individual with CML. A chromosomal preparation of cells at the metaphase stage of mitosis was stained with Giemsa, which binds A-T base pairs. The Philadelphia chromosome (the minute, defective chromosome 22 indicated by the right arrow) results from reciprocal translocation between chromosomes 9 and 22. Left arrow indicates portion of chromosome 22 translocated to chromosome 9 . Figure reproduced with permission from eMedicine.com (26).

first year of my pathology residency with Philip Custer in Philadelphia (from 1953 to 1954), we had focused primarily on hematopoietic neoplasms. In the Pathology Department of the University of Pennsylvania School of Medicine, I used a short-term cell culture technique developed by Edwin Osgood and Marion Krippaehne (10) to study human leukemic cells. The cells were grown on small slides, and I rinsed the cells with tap water before staining them with Giemsa to visualize their chromosomes. This was an inadvertent use of the "hypotonic technique" to disrupt the mitotic spindle and expand the cells, and it resulted in the presence of countable chromosomes in my metaphase chromosomal preparations. I knew nothing about cytogenetics at this time but felt that the chromosomal preparations of the leukemic cells warranted investigation for any abnormalities. I found no one on our campus interested in human chromosomes but was eventually directed to a graduate student, David Hungerford, who was working at the Fox Chase Cancer Center and attempting to obtain material for a thesis on human chromosomes.

Hungerford and I, as well as other researchers around the world, began to use the new cytogenetic techniques to determine whether human leukemias could be characterized by specific chromosome abnormalities. Although we initially found no consistent genetic abnormalities in cells from individuals with acute myelogenous leukemia, Hungerford identified a characteristic small chromosome in the neoplastic cells of two patients with chronic myelogenous leukemia (CML) (Figure 1) (11). We then began, with the help of Paul Moorhead, a scientist at the Wistar Institute, to use an improved air drying technique for the cell preparations, which had been developed by Rothfels and Siminovitch (12), and were able to report that a series of seven patients that we had analyzed all had this minute chromosome (13). Tough and colleagues (14), who were also studying human leukemias, designated this minute chromosome the "Philadelphia chromosome," in accord with the Committee for the Standardization of Chromosomes, which had suggested that abnormal chromosomes be named for the city in which they were discovered.

Our observation that all the neoplastic cells in nearly all cases of a specific human cancer contained a consistent somatic genetic change provided strong evidence to support Boveri's hypothesis (2) that a critical genetic alteration in a single cell, which provided the cell with a growth advantage, could give rise to a tumor. However, in the years immediately before and after the identification of the Philadelphia chromosome, consistent chromosomal alterations were not found in other types of leukemia. The only other apparent consistent alteration, which was noted in a number of patients with chronic lymphocytic leukemia in New Zealand and designated the Christchurch chromosome (15), proved not to be a somatic alteration but rather a familial abnormality in one particular family in that area.

\section{Chromosomal abnormalities in other cancers: result, not cause, of the tumor}

During the 1960s, analysis of some human solid tumors revealed that in nearly all types of cancer the chromosome pattern was abnormal (16). In particular, extensive chromosome alterations, such as chromosome numbers in the hypotetraploid range and gross structural chromosome rearrangements, were observed in individuals with very advanced tumors and malignant effusions. Furthermore, the extent of the cytogenetic changes often correlated directly with the extent to which the tumor had progressed clinically (17) - as had been observed earlier in experimental tumors that caused the accumulation of tumor-derived fluid in the abdomen (ascites tumors) - with the tumor consisting of a single stem line of cells or several closely related sub-lines. However, the presence of a consistent chromosome abnormality in all neoplastic cells was somewhat less common in solid neoplasms than in the leukemias, and in fact, huge variety in the number and type of chromosome alterations in a given tumor were often observed (17). Most importantly, when stem lines were present in solid tumors, they typically differed in their chromosome abnormality from one individual to another. Indeed, although a proportion of cases of some types of tumors, including tumors of the ovaries, testes, and meninges (18), show a characteristic chromosome abnormality, these abnormalities are not found in sufficient individuals with a given type of tumor to be considered a marker chromosome for the neoplasm. Thus, no chromosomal change comparable in consistency to the Philadelphia chromosome has been observed.

Part of the difficulty of identifying genetic abnormalities characteristic of specific neoplasms was methodological. Although during the 1960s technical advancements continued to be made - for example, Hungerford and coworkers developed more efficient hypotonic solution methods for generating chromosome preparations, and phytohemagglutin was used to stimulate mitotic cell division in lymphocyte cultures, thereby providing an easy source of mitotic chromosomes from non-neoplastic cells (19) - it was still impossible to individually identify human chromosomes. Furthermore, the often poor technical quality of metaphase chromosomal preparations from tumor material, as opposed to normal cells, made even accurate counting of chromosomes sometimes difficult.

Despite the lack of progress in identifying genetic abnormalities characteristic of specific neoplasms during the 1960s, other areas of research provided evidence to support the concept that chromosome abnormalities are associated with cancer. An increased number of spontaneous chromosome breakages were observed to occur when chromosome preparations were made from circulating normal lymphocytes that had been isolated from individuals with inherited clinical disorders associated with an increased risk of leukemia and other malignancies such as Bloom syndrome, ataxia 
telangiectasia, and Fanconi anemia. This association between an increased risk of developing tumors and increased chromosomal breakage led to the suggestion that chromosomal breakage might be an important tumorigenic factor (20).

Taken together, the data discussed here and elsewhere (21) that was generated by the end of 1960s led to the general opinion that most human tumors were associated with chromosome alterations and that the more advanced a neoplasm was the more extensive the chromosome alterations were likely to be. However, because no consistent chromosome abnormality other than the Philadelphia chromosome had been associated with a specific type of tumor, it was thought that the chromosome alterations were probably the result, rather than the cause, of the neoplasm. Furthermore, although some investigators thought that the chromosome abnormalities had an important role in tumor progression, others believed that they had no basic role in tumorigenesis.

\section{What happened next ...}

It must be remembered that in this era the field theory of tumor development, i.e., the theory that neoplasms arose from many cells in a tissue made susceptible by exposure to carcinogenic agents, was still widely believed. It was also a time when most investigators did not think that tumors were caused by genetic mutations. This might, in part, have been a reflection of the hope, more emotional than scientific, that tumors did not arise from structural changes in the genome because if this was the case, they would not be easy to reverse and treat. It was not until the 1970s that cytogenetic banding techniques and other methods were finally developed that generated more specific evidence at the level of individual chromosomes for some of the mutational hypotheses originally investigated by Boveri (2), and ultimately this led to the development of molecular tech- niques that permitted the identification of the specific genes altered not only in CML but in many hematopoietic and solid tumors.

As is discussed in detail in other articles in this Review series, subsequent developments with respect to the Philadelphia chromosome, although they have taken nearly 50 years, have been successful in that we have been able to use our knowledge to find specific targets and specific therapies for human cancer. In the 1970s, the improved cytogenetic techniques demonstrated that the Philadelphia chromosome resulted from a translocation between chromosomes 9 and 22 (Figure 1) (22), and later molecular techniques identified the critical genes involved as $\mathrm{v}$-abl Abelson murine leukemia viral oncogene homolog $(A B L)$ on chromosome 9 and breakpoint cluster region $(B C R)$ on chromosome 22 (23). Subsequently it was shown that the product of the $B C R-A B L$ fusion was an abnormal kinase that apparently was the stimulant for proliferation of myeloid cells to produce CML (24). Most recently, this kinase has been targeted by a newly developed therapeutic agent, imatinib mesylate (Gleevec), which has proved to have major positive therapeutic effects in patients with CML (25). These developments are still underway both for CML and for various other human neoplasms in which the specific altered genes have been identified. It is very gratifying to have participated in the early stages of this work and to see BCR-ABL, the product of the Philadelphia chromosome, as one of the first and best documented successful targets for an entirely new approach to cancer therapy.

Address correspondence to: Peter C. Nowell, University of Pennsylvania, M163 John Morgan Building, 36th Street and Hamilton Walk, Philadelphia, Pennsylvania 19104-6082, USA. Phone: (215) 898-8061; Fax: (215) 898-4227; E-mail: nowell@mail.med.upenn.edu.
1. Von Hansemann, D. 1890. Ueber asymmetrische Zelltheilung in Epithelhresbsen und deren biologische bedeutung. Virchows Arch. A Pathol. Anat. 119:299-326.

2. Boveri, T. 1914. Zur Frage der Entstehung maligner Tumoren. Gustav Fischer. Jena, Germany. 64 pp.

3. Painter, T.S. 1921. The Y-chromosome in mammals. Science. 53:503-504.

4. Winge, O. 1930. Zytologische Untersuchungen uber die Natur maligner tumoren. II. Tecrkarzinome bei Mausen. Z. Zellforsch. Mikrosk. Anat. 10:683-735.

5. Makino, S., and Sasaki, M.S. 1964. A chromosomal abnormality in a myelocytic aleukaemic leukaemia. Lancet. 42:851-852.

6. Hsu, T.C. 1979. Human and mammalian cytogenetics: an historical perspective. Springer. New York, New York, USA. 186 pp.

7. Harper, P.S. 2006. The discovery of the human chromosome number in Lund, 1955-1956. Hum. Genet. 119:226-232.

8. Ford, C.E., and Hamerton, J.L. 1956. The chromosomes of man. Nature. 178:1020-1023.

9. Bayreuther, K. 1960. Chromosomes in primary neoplastic growth. Nature. 186:6-9.

10. Osgood, E.E., and Krippaehne, M.L. 1955. The gradient tissue culture method. Exp. Cell Res. 9:116-127.
11. Nowell, P., and Hungerford, D. 1960. A minute chromosome in human chronic granulocytic leukemia [abstract]. Science. 132:1497.

12. Rothfels, K.H., and Siminovitch, L. 1958. An air-drying technique for flattening chromosomes in mammalian cells grown in vitro. Stain Technol. 33:73-77.

13. Nowell, P.C., and Hungerford, D.A. 1961. Chromosome studies in human leukemia. II. Chronic granulocytic leukemia. J. Natl. Cancer Inst. 27:1013-1035

14. Tough, I.M., et al. 1961. Cytogenetic studies in chronic myeloid leukaemia and acute leukaemia associated with monogolism. Lancet. 1:411-417.

15. Gunz, F.W., Fitzgerald, P.H., and Adans, A. 1962. An abnormal chromosome in chronic lymphocytic leukaemia. Br. Med.J. 2:1097-1099.

16. Sandberg, A.A. 1966. The chromosomes and causation of human cancer and leukemia. Cancer Res. 269:2064-2081.

17. DeGrouchy, J. 1967. Chromosomes in neoplastic tissues. Proceedings of the Third International Congress of Human Genetics. September 5-10, 1966. Chicago, Illinois, USA. J.F. Crow and J.E. Neel, editors. Johns Hopkins University Press. Baltimore, Maryland, USA.

18. Atkin, N.B., Baker, M.C., and Wilson, S. 1967. Stemlike karyotypes of 4 carcinomas of the cervix uteri.
Am. J. Obstet. Gynecol. 99:506-514.

19. Moorhead, P.S., et al. 1960. Chromosome preparations of leukocytes cultured from human peripheral blood. Exp. Cell Res. 20:613-616.

20. Harnden, D.G. 1974. Ataxia telangiectasia syndrome: cytogenetic cancer aspects. In Chromosomes and cancer. J. German, editor. John Wiley. New York, New York, USA. 619-636.

21. Nowell, P.C. 1993. Chromosomes and cancer: the evolution of an idea. Adv. Cancer Res. 62:1-17.

22. Rowley, J.D. 1980. Ph1-positive leukaemia, including chronic myelogenous leukaemia. Clin. Haematol. 9:55-86.

23. Groffen, J., et al. 1984. Philadelphia chromosomal breakpoints are clustered within a limited region, bcr, on chromosome 22. Cell. 36:93-99.

24. Lugo, T.G., Pendergast, A.M., Muller, A.J., and Witte, O.N. 1990. Tyrosine kinase activity and transformation potency of bcr-abl oncogene products. Science. 247:1079-1082.

25. Drucker, B.J., et al. 1999. Clinical efficacy and safety of an Abl specific tyrosine kinase inhibitor as targeted therapy for chronic myelogeneous leukemia. Blood. 94(Suppl. 1):A1639.

26. Besa, E.C., and Woermann, U. 2006. Chronic myelogenous leukemia. http://www.emedicine. $\mathrm{com} / \mathrm{med} /$ topic371.htm. 